\title{
Acumulación tóxica y despojo agroalimentario en La Mojana, Caribe colombiano
}

\author{
Toxic Accumulation and Agrifood Dispossession \\ in La Mojana, Colombian Caribbean
}

\author{
Juana Camacho S. \\ Instituto Colombiano de Antropología e Historia \\ jcamacho@icanh.gov.co
}

\begin{abstract}
RESUMEN
Este artículo aborda el vínculo entre contaminación y despojo en relación con el cultivo de arroz comercial en La Mojana, en el Caribe colombiano. Por su riqueza ambiental y productividad agrícola, esta región se ha proyectado como una despensa alimentaria. El arroz es el eje del sistema productivo, alimentario e identitario regional. A partir de un estudio etnográfico se examinan los efectos despojadores del uso de agroquímicos tóxicos en los medios de vida, la alimentación y la salud. Un argumento central es que, en su operación silenciosa y cotidiana, la contaminación vulnera bienes comunes y sistemas agroalimentarios locales, a la vez que refuerza otras formas históricas de despojo e inequidad social. Sin embargo, en pequeña escala, también existen otros modelos y prácticas locales que expresan alternativas en los tres escenarios propuestos: producción, dieta e identidad.
\end{abstract}

Palabras clave: sistema agroalimentario, acumulación tóxica, despojo, Caribe.

\begin{abstract}
This article discusses the link between pollution and dispossession related to commercial rice cultivation in the Mojana, Colombian Caribbean. Due to its environmental richness and agricultural productivity, this region has been posited as a food pantry. Rice is at the center of the regional productive system, diet, and identity. Based on an ethnographic study, I examine the dispossessing effects of toxic agrochemical use on livelihoods, food, and health. A central argument is that in its silent and daily operation, pollution degrades local commons and agrifood systems while reinforcing other historical forms of dispossession and social inequity. However, at a small scale, other local models and practices express alternatives in the three scenarios explored: production, diet, and identity.
\end{abstract}

Keywords: agrifood system, toxic accumulation, dispossession, Caribbean. 


\section{Introducción'}

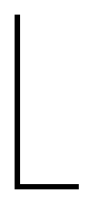

a alimentación y la salud dependen en parte del acceso a bienes y recursos ligados a la naturaleza. El aire, el agua, la tierra, los bosques, las plantas y los animales silvestres son bienes comunes, en tanto recursos y procesos naturales que pertenecen o producen beneficios a una comunidad y hacen posible su reproducción (Nonini 2007, 1; Ostrom 2000). Pero cada vez más tales espacios y recursos se mercantilizan y privatizan debido a su valoración y regulación en función de la productividad y el interés económico. Con ello no solo se debilitan los variados sistemas de uso, tenencia y manejo de estos bienes por parte de las comunidades, sino que se ponen en riesgo sus medios de vida, su seguridad, su diversidad y autonomía alimentaria $^{2}$. De allí que el cercamiento, la privatización y mercantilización de espacios y bienes comunes rurales sean temas de debate y movilización social contra el despojo contemporáneo (Linebaugh 2008).

En su definición clásica, el despojo se ha conceptualizado como un resultado de las relaciones sociales de poder y propiedad, donde los agentes del capital o el Estado privatizan y separan a los productores de los medios de producción para la acumulación (De Angelis 2001; Marx [1867] 1976). Más recientemente se ha vinculado con el cerramiento y la privatización de los bienes comunes y el desmantelamiento de los derechos de acceso y usufructo de las comunidades a los medios de subsistencia, mediante distintas formas de violencia sobre la gente y la naturaleza (Polanyi [1944] 1991; Zibechi y Hardt 2013), lo que también se ha denominado acumulación por desposesión en el contexto neoliberal (Harvey 2003). En los últimos años, el despojo se ha convertido en un importante campo de teorización y denuncia de la concentración y el acaparamiento global de tierras (Borras et al. 2011; White et al. 2012), el cerramiento de bosques y aguas (Fairhead, Leach y Scoones 2012; Kay y Franco 2012) y la vulneración de la alimentación y

1 La base documental de este artículo proviene de una investigación etnográfica realizada entre el 2013 y el 2015 en los municipios de San Marcos y San Benito Abad (Sucre); incluye entrevistas con agricultores y pescadores locales, agrónomos, técnicos y funcionarios municipales, departamentales y nacionales. Agradezco a todos ellos por su colaboración, al igual que a Nurys Silva, Julio Arias Vanegas, Alhena Caicedo Fernández y a los evaluadores por sus comentarios y sugerencias.

2 La seguridad alimentaria se define como la disponibilidad y el acceso físico, social y económico permanente, y en cantidad suficiente, a alimentos inocuos, nutritivos y culturalmente aceptables, para llevar una vida activa y saludable. La diversidad alimentaria se refiere a la variedad de alimentos consumidos, lo cual sirve de referente del acceso a nutrientes y la calidad de la dieta de una persona. Por autonomía alimentaria entiendo la capacidad de los pequeños productores de decidir sobre sus propios sistemas de producción para su sustento sin depender de otros e independientemente de las políticas nacionales alimentarias. 
la salud (Ezquerro-Cañete 2016). En Colombia se ha vinculado primordialmente con la concentración histórica de la tierra, el conflicto agrario y el control territorial (CMH 2010; CNRR 2009) y, más recientemente, con el extractivismo33.

Ciertamente, el despojo es un proceso complejo, continuo y multidimensional, no reducible al daño físico o material por la privación de la propiedad o el acceso a un recurso, sino que tiene implicaciones psicológicas y morales al fracturar relaciones sociales y vínculos afectivos y simbólicos con lugares y paisajes (Butler y Athanasiou 2013). No obstante, el despojo no siempre es explícito o evidente; en ocasiones ocurre de maneras invisibles y silenciosas mediante procesos que se naturalizan en la vida cotidiana, al igual que sus efectos corrosivos en personas y ambientes. Irónicamente, el despojo puede emanar de acciones justificadas en nombre del bienestar o del mejoramiento de las condiciones de vida (Li 2007), o que realizan quienes en últimas son sus víctimas. Tal es la situación de la contaminación por insumos tóxicos en la producción agrícola moderna, cuyo uso se sustenta en la racionalización y eficiencia productiva, la rentabilidad económica, la seguridad alimentaria y la salud, y cuyos efectos nocivos recaen directamente en los agricultores, trabajadores del agro y los consumidores.

El sistema agroalimentario moderno ${ }^{4}$ es uno de los principales agentes de deforestación, contaminación de suelos, aguas y aire, emisiones de gases de efecto invernadero, así como de la simplificación de la diversidad biológica y agrícola. Los insumos de síntesis química alteran procesos ecológicos y la cadena trófica en ecosistemas terrestres y acuáticos con consecuencias para la salud humana (Convenio de Estocolmo 2001). La contaminación ocurre en un doble movimiento de dispersión y concentración que magnifica sus efectos negativos. Por una parte, las sustancias tóxicas fluyen y se irradian por bienes comunes de la naturaleza, como aguas, aire y biodiversidad. Por otra, se acumulan en territorios, medios de subsistencia, alimentos y organismos humanos y no humanos. En esta doble vía, la contaminación se proyecta desde escalas ecosistémicas hasta las microgeografías íntimas del cuerpo, vulnerando la vida en sus múltiples expresiones. Así, despoja a las comunidades rurales de los recursos necesarios para pologías del despojo en Colombia I" de la Revista Colombiana de Antropología 52-2, 2016.

4 El sistema agroalimentario moderno se caracteriza por la industrialización del proceso productivo mediante la incorporación intensiva de capital, ciencia y tecnología; la integración del proceso de producción, distribución y consumo en cadenas alimentarias globales; la mercantilización de la comida como un factor de acumulación de riqueza y su concentración por parte de corporaciones transnacionales (Patel 2008). 
la producción y reproducción de la vida y socava los derechos fundamentales, al ambiente sano, el agua, la alimentación y la salud ${ }^{5}$.

Como lo señala Perreault (2013), la particularidad del despojo por contaminación es que opera mediante externalidades negativas del sistema de producción; es decir, transfiere los costos y efectos sociales y ambientales a la naturaleza, a las víctimas y a las generaciones futuras. Este despojo funciona por mecanismos extraeconómicos, pues no parte de la acumulación de la tierra, de los medios de producción, o de la fuerza de trabajo, sino de la transformación y degradación de los procesos y relaciones biofísicas que afectan la productividad, la fertilidad de los suelos, la calidad del agua, la inocuidad alimentaria, la salud, entre otros (Perreault 2013, 1065). No obstante, la contaminación sí tiene efectos económicos: por un lado, genera valor y permite la acumulación de capital de la industria agroquímica y agroalimentaria, como respuesta a acciones estatales y privadas dirigidas a maximizar la productividad y la rentabilidad ${ }^{6}$. Por el otro, la acumulación tóxica en los ambientes y los cuerpos separa la mano de obra de los habitantes y trabajadores rurales de sus medios de existencia y de la salud, con lo cual exacerba otras formas de despojo a la vez que profundiza la exclusión económica y social, como veremos.

En la lógica de "más alimentos a menor precio", la acumulación de capital se logra no solo a través de la renta de la naturaleza y del encerramiento de recursos y bienes comunes, sino de lo que Mitman, Murphy y Sellers (2004) llaman paisajes expuestos, para referirse a los lugares y cuerpos donde convergen patógenos naturales y agentes tóxicos de la química moderna que degradan los procesos materiales y sociales de reproducción de la vida. De estas múltiples interacciones entre cuerpos, ambientes y variables sociopolíticas surgen nuevas biologías locales y se producen otras formas de corporización (embodiment) de la enfermedad (Auyero y Swistun 2009; Lock y Nguyen 2010), que inciden en la manera como los sujetos reciben la toxicidad, su respuesta inmune, la susceptibilidad a la enfermedad o al dolor, y la capacidad de recuperación en el corto, mediano y largo plazo.

Además de los derechos consignados en la Constitución Política de Colombia, el país se rige por normatividad derivada de la suscripción de convenciones y pactos internacionales, como el Convenio de Estocolmo sobre los Contaminantes Orgánicos Persistentes (COP), que se firmó en el 2001 con el objetivo de regular el uso de sustancias químicas tóxicas, resistentes a la degradación y que se acumulan en los ecosistemas terrestres y acuáticos, tales como pesticidas, $\mathrm{PCB}$, dioxinas y furanos.

6 A propósito de la acumulación por contaminación véase http://www.ejolt.org/2015/02/accumulation-contamination/. 
Este artículo aborda la relación entre modernización productiva y despojo en La Mojana, un gran paisaje de humedales, extensas pasturas y parches de bosque tropical del Caribe colombiano. En concreto, examina la contaminación producida por el uso de sustancias tóxicas en el cultivo comercial de arroz mecanizado y sus efectos despojadores sobre tierras, aguas y cuerpos. El arroz es el eje de la dieta, la seguridad alimentaria, la cultura y la identidad en esta región; se emplea como bien de uso, compra e intercambio. Históricamente se cultivaban arroces criollos de manera manual aprovechando la fertilidad natural de los suelos, pero desde la década de los setenta y con el propósito de convertir a La Mojana en una despensa alimentaria con el arroz como cultivo bandera, se impulsó la producción mecanizada con variedades mejoradas. Junto con la técnica llegaron los agroquímicos y, con ellos, las consecuencias nocivas para la salud humana y ambiental.

El argumento que propongo es que, de manera silenciosa, sutil y cotidiana, la contaminación se constituye en un despojo progresivo que se naturaliza en los discursos y prácticas de quienes emplean los insumos tóxicos en su labor, a la vez que permanece parcialmente oculta frente a la opinión pública y las autoridades ambientales y de salud. Esta forma de despojo no solo vulnera los patrimonios ambientales y agroalimentarios, sino que refuerza otras formas históricas de despojo, exacerba la desigualdad estructural y compromete la capacidad de reproducción de la vida. Las respuestas de los productores oscilan entre la resistencia y la aceptación debido a la ignorancia tóxica (Saxton 2014), las condiciones ambientales de La Mojana, la dinámica (geográfica, bioquímica, económica, cultural) de los agroquímicos y las prácticas agrícolas locales. Sin embargo, a pesar de la persistencia de la siembra tecnificada y el acomodamiento a los impactos degradantes de los agroquímicos, también existen otros modelos productivos y culturales que funcionan, en pequeña escala, como una suerte de "contranarrativa" agroalimentaria.

La estructura del texto es la siguiente: a continuación se describe La Mojana como una región cuyas particularidades ambientales favorecen la producción agropecuaria y la seguridad alimentaria local, pero también el histórico despojo y la inequidad social. Luego se discute el papel de las políticas de modernización y desarrollo en la degradación de los medios de vida que hacen posible la reproducción física y social de los productores de La Mojana y la manera como el despojo por contaminación se ignora, normaliza o resiste por parte de los agricultores, quienes son los agentes directos que la producen y la reciben. Enseguida se analiza el arroz en relación con la dieta y la cultura alimentaria y con otro modelo arrocero que tiene diferentes lógicas y remite al potencial de resistencia y mantenimiento de patrimonios materiales y culturales en la región. 


\section{La Mojana: tierra promisoria para el grano de la abundancia}

De aproximadamente 500.000 ha, La Mojana es un reservorio de biodiversidad nativa e introducida, doméstica y silvestre. Esta gran llanura inundable está delimitada por los ríos Magdalena, Cauca y San Jorge ${ }^{7}$ que drenan allí sus aguas y la convierten en un lugar de singular importancia ecológica, económica y alimentaria. Los ecosistemas acuáticos y terrestres son la base de la agricultura, la ganadería y la pesca, que son los principales renglones económicos con el 90\% del área productiva (Martínez 2013). En medio de esta diversidad y abundancia, la concentración de la tierra y la riqueza coexisten con altos niveles de pobreza, necesidades básicas insatisfechas, desempleo e inseguridad alimentaria (COMTS 2013).

La fertilidad natural de los suelos, la abundancia de agua, la lluvia y el brillo solar en La Mojana son particularmente ventajosos para el cultivo del arroz. Este cereal se introdujo en la Colonia al Caribe, donde fue cultivado por propietarios menores, aparceros y colonos en pequeña escala y en tierras baldías. Se sembraba y procesaba manualmente, sin abonos químicos, con poca mano de obra y capital, para el autoconsumo y la venta regional (Flórez 2012; Posada 1998) ${ }^{8}$. En sus prácticas cotidianas los agricultores manipularon las semillas y produjeron nuevas variedades, según las condiciones del entorno y las necesidades productivas. Desde mediados del siglo XIX, junto con la caña, el arroz fue el cultivo comercial más promisorio de La Mojana, por lo cual en 1870 la Junta Central de Agricultura de Bolívar ${ }^{9}$ contempló su tecnificación para competir en los mercados internacionales (Posada 1998). Aunque la producción siempre estaba expuesta al riesgo de inundación, sequías y plagas, Majagual, Achí y Sucre eran las áreas más arroceras y abastecían el puerto de Magangué, eje comercial entre el interior del país y el océano Atlántico.

7 El territorio de La Mojana abarca porciones de cuatro departamentos: Sucre (72\%), Bolívar (16\%), Córdoba (10\%) y Antioquia (2\%) y once municipios, en un área aproximada de $500.000 \mathrm{~km}^{2}$. Tiene una población de 390.417 habitantes, de los cuales la mitad son rurales (Ramírez 2012).

$8 \quad$ El arroz introducido por los españoles fue la variedad asiática Oryza sativa. Se cultivó desde el siglo XVI en Mariquita, Tolima, y en los siglos XVII y XVIII había siembras en el Valle del Cauca y Antioquia. Su presencia en Cundinamarca, los Llanos y la cuenca del río Atrato en el Pacífico se dio en los siglos XIX y XX.

9 La Mojana fue parte del departamento de Bolívar hasta la disolución de este en tres departamentos: Córdoba (1952), Sucre (1966) y Bolívar. 
La introducción de maquinaria, riego artificial y algunos fertilizantes en la primera mitad del siglo XX favoreció la expansión del cereal en el Caribe y en 1930 el 50\% de la producción nacional estaba concentrada en el departamento de Bolívar (Kalmanovitz y López 2002). Esta tendencia modernizadora se intensificó con las recomendaciones de la Misión Currie en 1950 y con la adopción del modelo de la Revolución Verde. El imperativo productivo era parte de este proyecto técnico, político e ideológico que pretendía dinamizar el sector agroalimentario e incrementar la producción global de fuentes calóricas, especialmente de cereales como el arroz, para erradicar el hambre y la desnutrición en países pobres (Escobar 1995) ${ }^{10}$. Efectivamente, la inversión de capital, ciencia y tecnología logró un aumento sustancial en la disponibilidad alimentaria nacional, y en particular del arroz, un alimento de bajo costo, rendidor y agradable al paladar que conquistó el estómago y el corazón de los colombianos hasta convertirse en eje de la dieta nacional y símbolo del consumo moderno (Camacho 2011).

Al igual que en otras zonas del país, la productividad arrocera en La Mojana se benefició del uso intensivo de herbicidas y fertilizantes sintéticos en los años setenta. Para entonces, Colombia era uno de los mayores consumidores de agroquímicos en América Latina, ya que el arroz y el sorgo empleaban el $60 \%$ de los fungicidas líquidos y el $40 \%$ de los fertilizantes (Suárez 2007, 37), tendencia que se mantuvo en los años ochenta, cuando aumentó el uso de herbicidas (Bellotti, Cardona y Lapointe 1990). Actualmente, Colombia es el segundo consumidor de plaguicidas en América Latina, con 16,7 kg por hectárea (Ideam 2015). De suerte que lo que se inició como una novedad de la agricultura moderna generó una dependencia de sustancias que presentan riesgo y daños crónicos en la gente y el ecosistema. En La Mojana, la contracara del auge productivo ha sido el deterioro gradual de la base natural y el consecuente despojo de los medios materiales y los procesos socioambientales de los que dependen las comunidades para su subsistencia.

\section{Intensificación productiva y bienes comunes}

A diferencia de la ganadería que históricamente ha sido el símbolo de poder económico y político y de prestigio social en La Mojana, la agricultura ha estado

\footnotetext{
10 Para un análisis de la Revolución Verde desde una perspectiva de larga duración y en relación con procesos de acumulación capitalista, reconfiguración estatal, cambio agrícola y despojo, véase Patel (2013).
} 
"un escalón más abajo"11. Sin embargo, con el impulso dado al arroz a partir de los años setenta, grandes, medianos y pequeños productores se volcaron a la siembra comercial del grano en busca de opciones productivas y de trabajo ${ }^{12}$. En pocos años, el arroz activó la economía regional, que hasta entonces había sido mayoritariamente de subsistencia, al generar nuevas fuentes de empleo e ingreso en los distintos eslabones de la cadena (producción, distribución, molinería y venta), estimular la migración de gente de otras regiones, dinamizar el mercado de tierras y contribuir a la acumulación de capital económico, social y político. Incluso, un técnico agrícola que llegó a La Mojana en pleno auge arrocero comenta que ciertas élites terratenientes y ganaderas vieron en el arroz una amenaza porque “dañó la plaza”, al mejorar las opciones laborales de los campesinos que trabajaban en condiciones de explotación ${ }^{13}$.

Con las nuevas prácticas productivas también se generaron transformaciones en los ecosistemas y los recursos de uso común. Como lo señalara un gran arrocero y político local en una entrevista:

En los setenta y ochenta se produjo mucho arroz acá; el arroz nos dio mucha plata pero nos dañó la cabeza porque siempre ambicionábamos más; nos llevó a tumbar monte, secar las ciénagas, voliar venenos, meternos en política y vainas.

A lo que se refiere este productor es a que el auge del arroz incentivó la apropiación ilegal de tierras baldías, el desmonte de áreas forestales y relictos de bosque, la desecación de humedales, la alteración de fuentes hídricas, la

11 Históricamente la ganadería de doble propósito ha sido más importante en área y número de productores. Sin embargo, en el 2012, luego de las inundaciones prolongadas por la ola invernal (2010-2011), el $45 \%$ del área productiva total estaba en sistemas mixtos (pecuario y agrícola con predominio del arroz), el $20 \%$ en sistemas agrícolas, el $16 \%$ en pesca y el $7 \%$ era pecuario (Martínez 2013).

Por ser un cultivo semestral, los productores pueden anticipar los retornos de la primera cosecha y tomar decisiones antes de la segunda siembra: si se pierde una cosecha, es posible recuperarse en la siguiente.

La modernización del arroz coincidió con las grandes luchas campesinas por la tierra y las políticas de reforma agraria de los sesenta, setenta y ochenta del siglo XX, que se destacaron en el Caribe y redundaron en la adjudicación parcelaria a familias sin tierra y el establecimiento de proyectos productivos comunitarios, entre ellos de arroz. Aunque la inequitativa distribución de la propiedad no se alteró significativamente, estas medidas contribuyeron a la transformación de las tradicionales relaciones sociales de producción, la seguridad alimentaria, la generación de empleo y el ingreso campesino al mercado a través del arroz comercial. Por diversas razones, entre ellas la falta de apoyo estatal a los proyectos productivos, poco a poco algunos campesinos vendieron las tierras y terminaron de jornaleros nuevamente (Zamosc 1990). 
destrucción de restos arqueológicos prehispánicos por el aplanamiento del sue$10^{14}$ y el desplazamiento de variedades locales de arroz. La instauración de una nueva estética rural de un paisaje plano, homogéneo y sin monte se hizo a costa de la transformación y el despojo gradual de baldíos, humedales, zapales, playones y zonas ${ }^{15}$, espacios y recursos que son verdaderos subsidios naturales del sistema económico y, especialmente, de los pobres y sin tierra, como lo ilustra el siguiente testimonio de un campesino:

Esto acá primero era baldío con montaña, se sembraba poquito arroz criollo en tierras que los patrones daban en préstamo pa la cosecha y se devolvían sembradas con pasto para la ganadería. Cuando vino el arroz comercial, entonces eso lo mecanizaban, o sea lo araban, en época de verano, y esos pastos se morían por la resequedad... En el momento de la limpia, le fumigaban herbicida que mata todo lo sucio... En algunos lugares taponaron los caños y en otros los zapales se fueron secando.

En La Mojana, la privatización y el despojo de baldíos, tierras de uso comunal y aguas públicas no son nuevos. Por el contrario, su apropiación, legal e ilegal, tiene una historia larga vinculada a la ganadería trashumante entre las zonas altas de sabana y las tierras bajas, adonde se llevan las reses en el verano (CMH 2010; Fals Borda 2002; Reyes 1978). La existencia de humedales y pastos naturales en playones y ciénagas ha hecho que grandes ganaderos hayan buscado asegurar el dominio sobre aguas y tierras de uso comunal, mediante el cercamiento directo o la compra -en algunos casos ilegal y violenta-. En esta región, consuetudinariamente los humedales y las tierras alrededor de los cuerpos de agua se han empleado por estaciones, de forma individual o familiar, para la agricultura, la ganadería, la pesca y la cacería. A partir del uso regular y comunal de tales espacios se configuró el "derecho de ciénaga", derecho de facto que tiene un sustento cultural en dichas prácticas (Camacho 2015). Además, jurídicamente, estas tierras y aguas tienen un cuerpo normativo que las define y rige como bienes públicos que no pueden privatizarse y deben estar disponibles para

La Mojana tiene una gran infraestructura hidráulica precolombina consistente en un elaborado sistema de canales, camellones y plataformas de cultivo y vivienda para el manejo de las inundaciones, las sequías y la sedimentación.

15 Los baldíos son reservas territoriales del Estado y bienes públicos imprescriptibles pero adjudicables. Los zapales son ecosistemas de bosque inundable, hábitat de mamíferos, reptiles, anfibios y aves. Los playones son baldíos inundables; normativamente, son reservas territoriales del Estado y bienes públicos imprescriptibles e inadjudicables, pero se permite su uso individual o colectivo para la subsistencia por vecinos del lugar que no posean tierra (Ley 160 de 1994). Las zonas son las tierras a la orilla del río; por ley, hasta los $30 \mathrm{~m}$ son bienes del Estado inembargables e imprescriptibles, excepto si existen derechos adquiridos, pero también son de uso público y común. 
el aprovechamiento de los vecinos del lugar en actividades de subsistencia (véase la nota al pie n. ${ }^{0}$ 15; Incoder 2014). Sin embargo, el "derecho de ciénaga” ha sido empleado por los más poderosos para justificar su apropiación y concentración, con lo cual no solo se despojan los bienes materiales sino los derechos comunes de acceso y uso de estos recursos.

Con estos antecedentes, lo mencionado por el político arrocero citado cobra un nuevo sentido al revelar continuidades históricas en las prácticas de encerramiento, degradación y despojo de espacios y medios de sustento, que ocasionan perjuicios, especialmente para los más pobres. Así, a la privatización e individualización de bienes comunes y derechos colectivos por la ganadería trashumante se superpone un proceso semejante, pero esta vez asociado con la intensificación productiva del arroz mecanizado.

\section{El arroz secano mecanizado}

A raíz de la modernización del arroz en La Mojana, el cereal se convirtió en la columna vertebral del sistema productivo, la economía, la dieta y la identidad regional. Actualmente se cultiva allí cerca del $12 \%$ de la producción del país (entre 15.000 y 25.000 ha) en un ciclo anual de dos cosechas y en ciertas áreas, hasta de tres. La estructura productiva consiste en el arroz secano mecanizado (71\%) y secano manual (29\%) (Corpoica-INAT 2012). El primero se hace bajo un modelo tecnificado de monocultivo, para el mercado regional y nacional, mientras que el arroz manual se destina al mercado local, al consumo doméstico y para la semilla. La producción mecanizada con un uso intensivo de agroquímicos la hacen grandes cultivadores (entre 30 y 100 ha o más), medianos (entre 6 y 30 ha) y pequeños productores (1-5 ha). En cambio, el arroz manual, que utiliza pocos o ningún agroquímico, está en manos de agricultores de subsistencia (entre 0 y 3 ha).

A diferencia de los grandes ganaderos, que son a su vez terratenientes, los grandes cultivadores de arroz no necesariamente son propietarios de la tierra. Una parte considerable de la siembra se hace en predios alquilados, por contrato verbal semestral, y con pago en efectivo o con una parte de la cosecha. Rentar tierra para arroz es común entre grandes, medianos y pequeños propietarios, así como entre agricultores sin tierra, por varias razones: primero, para evitar la proliferación de patógenos, pues luego de un ciclo agrícola se requiere el descanso de los suelos y la rotación de los cultivos. Segundo, para reducir el riesgo de pérdida por inundaciones. Tercero, porque la inequidad en la propiedad de 
la tierra obliga a los pequeños propietarios y a los agricultores sin tierra a alquilar terrenos en cada cosecha. Finalmente, en los sistemas de producción mixtos (agrícola y ganadero) se alquilan predios diferentes a los de pastoreo para evitar la contaminación de los animales por los agroquímicos del arroz.

El uso de insumos químicos durante el ciclo agrícola es común entre todos los productores que cultivan arroces mejorados de forma mecanizada. La diferencia es que los grandes arroceros, que tienen mayor acceso a los medios de producción, al capital o al crédito para cubrir los costos laborales y de los insumos (semillas, fertilizantes y plaguicidas), aplican los fertilizantes y herbicidas hasta tres veces en una cosecha, mientras que, por razones económicas, los pequeños emplean dosis menores. Los agroquímicos se usan desde el inicio del ciclo cuando se aplican herbicidas como el glifosato para limpiar y "sellar la tierra”. A los 15 días de la siembra se adicionan urea, potasio y triple $15^{16}$ para el crecimiento y la preñez o la formación de la espiga, dado que, según los agricultores, los arroces mejorados "son exigentes y uno tiene que asistirlos bastante". A las dos semanas se aplican pesticidas a fin de proteger la espiga, operación que se repite a los dos meses, porque "los arroces chiquitos son más débiles y vulnerables" a causa de la mayor artificialización de los procesos agronómicos. Según el régimen de lluvias, que es indispensable para el llenado del grano, a los 90 o 95 días se hace el corte, se cosecha y se embulta el arroz paddy (que aún tiene la cascarilla y mucha humedad), para ser llevado al molino a secar, pesar y vender. En el cultivo queda la soca que vuelve a producir al mes y medio o dos meses, pero en menor cantidad, por lo que se aplican nuevamente abonos foliares o "llena grano". Toda esta operación se repite dos o tres veces al año según el número de cosechas. Luego, quienes pueden dejan descansar el terreno durante un año mientras crece pasto natural, pero quienes viven del arroz y no tienen más tierra o dinero para alquilar inician el ciclo nuevamente.

16 Fertilizantes minerales que tienen tres de los macronutrientes primarios: nitrógeno, fósforo y potasio, necesarios para el crecimiento y desarrollo de las plantas. Su nivel de riesgo es objeto de debate, especialmente por las altas dosis en que se aplica, que pueden ser contraproducentes para la salud humana y animal. 


\section{Los venenos de la vida: acumulación tóxica y de capital}

Los riesgos ambientales de la contaminación asociada con el arroz mecanizado en La Mojana no han pasado desapercibidos (Aguilera 2004; DNP-FAO 2003), sin que esto se haya traducido en acciones preventivas o de mitigación para contrarrestar sus efectos despojadores. Es decir, sus impactos degradantes en las condiciones biofísicas y los procesos ecológicos que afectan la fertilidad de los suelos, la calidad del agua, la inocuidad de los alimentos y la salud humana y ambien$\mathrm{tal}^{17}$. En otras palabras, el despojo de las condiciones que permiten la reproducción física y social de la vida. Entre técnicos, funcionarios y productores locales, el uso de agroquímicos genera reacciones mixtas. Por una parte, coinciden en la necesidad de emplear herbicidas, insecticidas y fungicidas para controlar las malezas y los patógenos, estandarizar la producción y aumentar la productividad, ya que la humedad y la temperatura, que son condiciones ideales para el grano, también son factores limitantes. Para contrarrestar los riesgos de corto plazo, los productores recurren a los gremios sectoriales, las casas de insumos y los molinos que ofrecen créditos, distribuyen las semillas certificadas y prescriben "las marcas más recientes” de agroquímicos. Los pequeños agricultores, además, apelan a los consejos de vecinos y al ensayo de fórmulas empíricas con sustancias variadas, como lo describe un fumigador de oficio:

Acá uno acostumbra a fumigar con la bomba manual de espalda. Aquí nadie nos ha dicho que tenemos que fumigar así o asá. Fumigamos desordenadamente, uno aplica lo que diga el agrónomo o lo que mande el dueño del cultivo, o lo que converse con el vecino. A veces se ligan unos químicos con otros, o sea que van mezclados, por eso a veces se intoxica el arroz y eso también va al agua.

Por otra parte, los técnicos y productores reconocen los riesgos de la aplicación de insumos tóxicos en mezclas o cocteles que incluyen hasta cuatro clases

17 A pesar de los intensos debates sobre el uso de insumos tóxicos en la agricultura, estos se justifican en nombre de las necesidades económicas, productivas y de seguridad alimentaria. Un ejemplo de la ambigüedad institucional al respecto y de la presión de los fabricantes de estas sustancias son los pronunciamientos contradictorios de la Organización Mundial de la Salud. En el 2015, la Agencia Internacional para la Investigación del Cáncer señaló la posible carcinogenidad del glifosato y otros pesticidas organofosforados (IARC-OMS 2015), pero en el 2016 indicó la improbabilidad de que el glifosato presente riesgo carcinogénico para los humanos a través de la dieta (FAO-OMS 2016). El glifosato es producido por Monsanto, una de las mayores transnacionales estadounidenses especializada en agroquímicos y biotecnología, recientemente adquirida por Bayer. 
diferentes de ellos, con poca o ninguna asistencia técnica, en cantidades inapropiadas, en épocas no adecuadas y sin mayor protección física. Desbalances en la química del suelo, ineficiencia en la absorción de la planta, desperdicio por escorrentía, aumento de problemas fitosanitarios por destrucción de los controladores naturales y resistencia a los venenos y daños irreversibles en los cultivos y el ecosistema son algunos efectos adversos. Los pesticidas más comunes son de alta peligrosidad (Ideam 2015, 276), en especial, los no biodegradables, que persisten en suelos y aguas y se acumulan en plantas, animales y alimentos ${ }^{18}$. En varias regiones del Caribe, la leche cruda con presencia de residuos de plaguicidas por encima de lo permitido proviene de regiones aledañas a zonas arroceras (Pongutá, Lans-Ceballos y Barrera-Violeth 2012; Solano y Marrugo 2014).

Para los habitantes de La Mojana, y en particular para los productores de arroz - grandes, medianos y pequeños-y los pescadores, los problemas por la contaminación tampoco pasan desapercibidos. Como lo mencionó un profesional de la salud y arrocero importante, mientras que los más ricos tratan de contrarrestar los problemas productivos y ambientales con mayor inversión económica, los más pequeños y más directamente afectados en su salud por la exposición directa desconocen muchas de las consecuencias tóxicas y no cuentan con recursos para mitigarlos. Por su parte, un pequeño agricultor comentó: "Hemos visto que el pescado que vive en las aguas cerca a los cultivos se muere después que se fumiga”, mientras que al momento de hacer el desayuno, la esposa de un pescador dijo que últimamente nota un sabor a flores en los peces y no sabe si es por los detergentes de lavar la ropa que se vierten a la ciénaga o por los venenos del arroz. Sobre los efectos en otros cultivos, un campesino añadió que las plantas que no se fumigan "se afectan con el viento o el mero olor del químico; la berenjena, el tomate y el pepino aspiran la contaminación y se doblegan”; y con respecto a los animales un trabajador mencionó:

A veces se saca el ganado si aplica veneno fuerte porque las vacas preñadas abortan por comer el pasto que queda, pero cuando uno fumiga y las vacas están en el potrero, dice uno que no les pasa nada porque no las ve caer...

En Colombia, de los plaguicidas empleados y aprobados para la venta, el 1,8\% son extremadamente peligrosos, el $23 \%$ son altamente peligrosos y el $47 \%$ tienen una peligrosidad moderada (Cárdenas et al. 2005, 2010). El Ministerio de Salud y el Instituto Colombiano Agropecuario (ICA) son los principales reguladores de los plaguicidas químicos y se rigen, entre otros, por la normatividad nacional (Ley 822 del 2003; Decreto 502 del 2003) y de la Comunidad Andina (Decisión 436 de 1998) sobre el registro y control de plaguicidas químicos de uso agrícola y su comercio en la subregión. Consultado el 12 de febrero del 2016. http://www.ica.gov.co/ getdoc/2dae6093-c021-49d1-8b29-c9dfebce2757/REGISTROS-DE-VENTA--PQA-24-01-09.aspx. 
Así, a pesar de la inquietud que pueda suscitar la contaminación, esta se naturaliza como parte del conjunto de hechos, incertidumbres y retos cotidianos de quienes viven en un paisaje de gran complejidad ecológica, dinamismo climático y riesgo ambiental. Lo anterior evoca lo planteado por Auyero y Swistun (2009) sobre "la confusión, los errores y la ceguera de la gente con la contaminación que los rodea” (4), y la manera silenciosa como se habitúan y acomodan a esta, o en algunos casos, reconocen la impotencia frente a las fuerzas del mercado y la exigencia de una mayor productividad.

Con las alteraciones del régimen climático y los patrones de lluvia, aumenta el uso de los agroquímicos y se agudizan las demandas del sector agropecuario y campesino al Estado para que controle y regule los precios de los insumos de síntesis química. Estos están entre los más caros de América Latina, pues son en su mayoría importados y muy vulnerables a las fluctuaciones del mercado ${ }^{19}$. Su comercio, además, está en manos de monopolios que definen el precio interno, entre el $30 \%$ y el $50 \%$ por encima del valor mundial (DNP 2009; Marín 2013). Este mercado mueve en el país alrededor de 1,5 millones de toneladas de fertilizantes que equivalen al $95 \%$ de las ventas totales, de las cuales un $94 \%$ se concentra en seis empresas (DNP 2009). Por ello existe un mercado de plaguicidas de contrabando, algunos prohibidos por su peligrosidad. La poca regulación sobre el uso indiscriminado y excesivo de los insumos, el elevado precio y su monopolio comercial apunta a la relación directa entre la acumulación de capital y la acumulación tóxica, entre otros factores ${ }^{20}$.

En estas condiciones, y de cara a la internacionalización de la agricultura, los discursos acerca de la competitividad nacional frente a países con producciones altamente subsidiadas, y con los cuales Colombia mantiene tratados de libre comercio, son estrategias retóricas que se contradicen con las circunstancias reales de muchos productores, en particular, los pequeños (Garay, Barberi y Cardona 2012; Suárez 2015). A su vez, la industria agroquímica y farmacéutica, los Gobiernos y sectores del estamento científico y médico minimizan los riesgos tóxicos con argumentos económicos, técnicos y morales, en especial cuando los más afectados son poblaciones y lugares estructuralmente vulnerables (Saxton 2014). A propósito, un agrónomo consultado, quien ratificó la necesidad de

19 Luego del Paro Agrario Nacional del 2013, el Gobierno colombiano expidió el Decreto 1988 que reglamenta la política de intervención de precios de insumos agropecuarios por parte del Ministerio de Agricultura y Desarrollo Rural, sin resultados efectivos hasta la fecha. la convergencia de tecnología y capital y la integración vertical, producen y controlan no solo los agroquímicos sino las semillas que se producen cada vez más con biotecnología: Bayer, Monsanto, DuPont, Syngenta, Dow y Basf. 
emplear agroquímicos para el control de plagas en el arroz comercial por ser este uno de los principales alimentos y fuentes de empleo e ingreso regional, también señaló que, si bien no son prácticas inocuas, la ecología del ecosistema (los humedales, la dinámica del agua, el clima, las inundaciones) y la abundancia de microorganismos del suelo que degradan los químicos reducen el riesgo tóxico y favorecen la rápida capacidad de recuperación ambiental ${ }^{21}$.

\section{Contaminación y despojo de la salud}

En el 2014, el 28,67\% de las intoxicaciones por sustancias químicas en Colombia fueron por plaguicidas (INS 2016) ${ }^{22}$. La exposición por inhalación o contacto directo afecta órganos, tejidos y células, y produce alteraciones bioquímicas, fisiológicas y morfológicas de corto y largo plazo que redundan en enormes costos sociales, económicos y de salud pública (Fernández, Mancipe y Fernández 2010; INS 2010). Durante la gestación y la lactancia, las mujeres pueden transmitir los agentes contaminantes a los hijos, de manera que el despojo por toxicidad ocurre no solo en los medios de vida y el ambiente inmediato, sino también en los cuerpos y la salud de las generaciones presentes y futuras.

Mareos, rasquiña, enrojecimiento de la piel, dolor de cabeza, desmayo, diarrea, calor y debilidad son algunos de los síntomas más comunes mencionados por los entrevistados en La Mojana. Los mezcladores y fumigadores de agroquímicos, los agricultores y trabajadores, en su mayoría hombres con bajos niveles de escolaridad, poco acceso al sistema de salud e información fragmentaria y contradictoria sobre los riesgos tóxicos, son los más directamente afectados. Por su participación en labores agrícolas las mujeres también están expuestas —“Mi esposa se intoxicó con el olor de la amina (herbicida), duró dos días hospitalizada y le duele la cabeza cuando aplican venenos"-, e indirectamente a través de oficios domésticos, como el lavado de ropa impregnada de químicos que puede producir mareos y rasquiña. La presencia de químicos en recintos cercanos a la

21 El uso de microorganismos, hongos o plantas para eliminar o neutralizar contaminantes o restaurar ambientes contaminados se conoce como biorremediación. Otro argumento esbozado es que La Mojana no presenta el mismo riesgo de contaminación si se compara con el Tolima y los Llanos, en donde la cantidad de agroquímicos, los costos de producción y la productividad se duplican. 
vivienda o dentro del hogar también constituye un riesgo para todos los miembros de la familia.

Pero, a semejanza de las percepciones y vivencias de la contaminación agrícola y ambiental, el despojo de la salud por la intoxicación directa e indirecta que produce daño y enfermedad también se naturaliza en el día a día, mientras el malestar no sea extremo: "la intoxicación, la rasquiña, el enrojecimiento de la piel se toman como cosas leves, son cosas del ambiente, reacciones normales del químico", comenta un joven agricultor. Para una población campesina cuyas labores requieren de gran resistencia física, templanza y adaptabilidad, estos son gajes del oficio a los que "no se les para bolas", en especial cuando se trata de los hombres mojaneros, simbolizados con la figura del "hombre hicotea” (Fals Borda 2002), por su fortaleza y capacidad de aguante frente a las duras condiciones ambientales y sociales a las que están expuestos. La conjunción de imaginarios y representaciones culturales, roles de género e ignorancia tóxica (Saxton 2014) también contribuye a la negación parcial o al no reconocimiento del daño, como se revela en las palabras de quien, como la hicotea, que se entierra en la época de sequía y va mudando la piel que la protege, pareciera tener un caparazón que se regenera con la adversidad:

Gracias a Dios no me he intoxicado. Uno por acá no usa máscara ni guante porque pesa y da calor, solo una camisa de manga larga y un trapo para taparse la boca. Cuando me mareo, me duele la cabeza o me irrito, me baño o tomo agua de panela. Si me cae un veneno fuerte que lo pone a uno amarillo, me echo tierra y espero que se caiga el cuero.

Cuando no se considera necesario ir al médico, se usan remedios caseros: baños con jabón, agua de panela para que el veneno "salga rápido del cuerpo", huevo o aceite para provocar vómito. Si esto no funciona, se acude al centro de salud o al hospital para recibir sueros que alivien los síntomas, pero la falta de dinero para el desplazamiento, la imposibilidad de perder un día de trabajo y la poca confianza en el personal sanitario limitan la consulta hospitalaria. De acuerdo con los funcionarios de salud municipales, departamentales y nacionales consultados, a pesar de la agenda nacional en salud ambiental, las políticas y planes de contaminación son deficientes en la identificación, caracterización, prevención y gestión del riesgo ${ }^{23}$. Médicos, enfermeras y funcionarios aducen

23 La regulación del uso y manejo de plaguicidas está consignada en la Ley 9 de 1979, la Resolución 04547 de 1998 y los decretos 1843 de 1991 y 3518 del 2006. http://www.ins.gov.co/tramitesy-servicios/programas-de-calidad/piccveo/Protocolo\%20Programa \%20VEO\%202015-vf200315. pdf. Para mayor información sobre la contaminación por pesticidas, véanse Cárdenas et al. (2005, 2010, 2012). 
que la información disponible y las acciones de vigilancia epidemiológica de plaguicidas $^{24}$ son limitadas por falencias en la captura de datos, la baja cobertura, la falta de recursos y la inexperiencia del personal de salud en el tema toxicológico. La sintomatología además se confunde con virosis, enfermedades parasitarias o bacterianas, que son las principales causas de morbilidad en la región, y que se exacerban con la pobreza, la malnutrición, la precariedad del saneamiento básico y el cambio climático.

Vemos entonces que por vías distintas a las prácticas históricas de despojo, asociadas con la apropiación y privatización de tierras y aguas públicas de uso comunal, la contaminación y acumulación tóxica también tienen efectos despojadores en los que se entretejen la ecología y economía política de los agroquímicos y del sistema agroalimentario con la micropolítica del cuerpo y la salud. Son formas sutiles y naturalizadas que exacerban la vulnerabilidad e inequidad histórica de gentes y territorios. En este sentido, la exposición a los pesticidas es un aspecto de la vulnerabilidad estructural de los agricultores (Quesada, Hart y Bourgois 2011) y de las incertidumbres en un sistema agroalimentario y de salud donde convergen actores con distintos intereses, percepciones, conocimientos y aproximaciones en relación con la toxicidad y el riesgo.

\section{Paradojas y posibilidades de una despensa alimentaria en el trópico}

La Mojana encarna una de las grandes paradojas de la modernización productiva. En el intento por hacer de este gran paisaje una gran despensa regional, los efectos acumulativos de la toxicidad en suelos, aguas, ecosistemas, animales y otros cultivos no solo vulneran la seguridad y sostenibilidad agroalimentaria, sino la salud humana y ambiental. Ahora bien, como se advirtió antes, lo que llama la atención y se constituye en una alternativa local y de pequeña escala a la hegemonía del imperativo productivista global y a la reconfiguración de los espacios y medios de vida locales es que, al lado de la agricultura empresarial

Para prevenir y monitorear los riesgos tóxicos, luego de una serie de intoxicaciones masivas en los años sesenta y setenta se creó el Programa de Vigilancia Epidemiológica de Plaguicidas (VEO). Las secretarías de salud realizan periódicamente análisis de colinesterasa -enzima asociada con el sistema nervioso que se inhibe con la presencia o exposición a organofosforados y carbamatos empleados en pesticidas- en individuos considerados en riesgo de intoxicación. 
mecanizada del arroz, se cultivan variedades criollas para el uso doméstico y en ocasiones para el mercado local en molinos y tiendas. Si bien los grandes y medianos arroceros siembran a veces una o dos hectáreas de variedades criollas, en general, son los pequeños agricultores y los sin tierra quienes, de forma manual, en pequeña escala y con poco o ningún uso de insumos químicos, los cultivan para su propio consumo durante el año ${ }^{25}$. ¿Por qué y cómo se mantiene un sistema que se rige por otras lógicas económicas, agronómicas y culturales, y que a la vez ofrece distintas opciones de seguridad y diversidad alimentaria y salud? Algunas claves de estas prácticas están en la cultura alimentaria, la identidad y la relación corporizada de los mojaneros con la comida.

Contrario a quienes opinan que los campesinos del Caribe carecen de refinamiento y “buen gusto” culinarios (Posada Carbó 1994) ${ }^{26}$, en La Mojana hay una rica y compleja cultura alimentaria, atravesada por la historia productiva, el uso y manejo del paisaje, y el aprendizaje social del gusto. En sus prácticas diarias de producción, preparación y consumo directo de los alimentos, que involucran directamente al cuerpo y los sentidos, los hombres y mujeres de La Mojana desarrollan sensibilidades con respecto al sabor, la textura, la apariencia y la calidad general de los cultivos, los animales y las comidas. Estas prácticas e imbricaciones materiales, sensoriales y emocionales con los alimentos configuran una relación corporizada (embodied) con la comida (Camacho 2011; Carolan 2011), que se forja en relaciones históricas, sociales y culturales más amplias (Farquhar 2002). En efecto, la dieta y la cocina de La Mojana son producto de la fusión de los sistemas agroalimentarios y culturales indígenas, europeos, negros y árabes que convergen en el Caribe.

A semejanza de otras sociedades agrarias, el repertorio alimentario mojanero es dinámico, sin embargo, la estructura cotidiana se basa en una tríada de productos locales: arroz, liga y bastimento o vitualla. El arroz es el centro del plato y eje del hábito alimentario regional; pobres y ricos lo consumen hasta tres veces al día; incluso hay quienes prefieren no comer si no hay arroz en el plato, porque "sin arroz no hay Dios”. Este alimento rendidor, de fácil almacenamiento y suave digestión tiene variados usos culinarios en preparaciones sólidas y

25 Los criollos equivalen aproximadamente al 10\% del total de la producción arrocera de La Mojana.

Como ejemplo de la valoración negativa de la dieta costeña, en 1881 el cónsul de Estados Unidos en Cartagena comentó que sin el plátano "en este país la gente no sabría cómo vivir bien", que del maíz preparaban una "masa indigerible llamada bollo" y el arroz era "el único plato que los nativos saben cocinar con propiedad" (Posada 1994, 249). Ideas semejantes se repiten en muchos sectores sociales con respecto a ciertos productos de la comida y cocina popular del Caribe. 
líquidas, de dulce y de sal: sopas, arroces de varios tipos, bollos, arepas, dulces y chichas. Un plato "pobre pero sabroso" es el arroz con queso, con suero o con vinagre. Por su centralidad en el sistema agroalimentario y la cocina regional, el arroz es emblemático de la relación corporizada con la comida.

La liga es la proteína animal, fuente de fuerza y energía que incluye carne de origen doméstico o silvestre, pescado, queso, suero y huevo. Los peces y carnes silvestres, vistos por algunas élites como "comidas de pobre", han sido claves en la seguridad y diversificación alimentaria de campesinos, pescadores y habitantes urbanos sin tierra. Plátano, yuca, ñame, ahuyama, batata y maíz componen el bastimento o la vitualla. Ingredientes como ajo, ají dulce, pimentón, cebolla y hierbas aromáticas son la base de la sazón "criolla costeña".

Comida se define como el alimento cocinado, de sal y fresco, es decir, preparado el mismo día pero no crudo ni congelado. El gusto por lo fresco y lo cocido se debe en parte a la falta de refrigeración y la necesidad de prevenir la rápida descomposición de los alimentos, aunque la salazón ha sido una forma de conservación de pescados, carnes y lácteos. El consumo de una porción abundante y en un horario regular se considera fundamental para la nutrición, pues al decir de la gente local, "comer bien no es jactarse ni comer de lujo, es que dé energía para el trabajo”. La composición del plato y la frecuencia de la ingesta varían según la disponibilidad alimentaria, la estacionalidad, la actividad que se esté realizando y el ingreso.

El consumo de alimentos limpios y sin químicos alude a la ausencia de insumos químicos y a la calidad deseable de la comida. Este principio de calidad se asocia con la comida criolla que, al igual que en otras regiones del país, se refiere a la comida y la cocina locales ${ }^{27}$. Como lo señaló un agricultor entrevistado:

El arroz criollo es necesario para la alimentación, sin el arroz el trabajador no tiene fuerza, no va más. Un ejemplo de buena comida es el arroz criollo, el huevo criollo, la leche fresca. Esta es comida buena, fresca, limpia, criolla, natural.

Los productos criollos, locales o regionales, con los cuales hay mayor familiaridad, permiten una suerte de trazabilidad con respecto al origen, las condiciones de producción, su frescura y sabor, así su producción no siempre sea orgánica.

Los arroces criollos son variedades introducidas a La Mojana desde hace varias generaciones por los agricultores y adaptadas a las condiciones locales. Se estima que allí existen cerca de 80 variedades derivadas de dos subespecies:

27 En su acepción original, criollo se empleó en la Colonia para denominar a los descendientes de europeos nacidos en América. En el caso de la comida, alude a los ingredientes, formas de cocción y sabores nativos de un lugar, que pueden tener influencia española o africana. 
japónica ( $77 \%$ ) e índica ( $23 \%$ ), con características particulares en el aspecto físico, el comportamiento vegetativo, el sabor y el costo, lo que hace de la región un centro de diversidad arrocera (Corpoica-INAT 2012). En las tierras altas predominan las variedades de secano, conocidas como finos, y en las tierras bajas inundables se cultivan las de agua, conocidas como chiles, chilimicos, chombos o arroces de los pobres. Las familias de menores recursos y los pequeños agricultores los cultivan en parcelas de entre 0 y 2 ha. Si bien algunas variedades crecen más lentamente y la productividad es menor, son apetecidas y valoradas al ser "más fuertes, resistentes a las plagas, y más pesados porque el grano tiene más leche”, ventaja importante a la hora de pesar el costal en los molinos donde se imponen el precio y las condiciones de compra ${ }^{28}$.

En el momento de la siembra, si el terreno ha estado en descanso y tiene rastrojo, se emplea el sistema tradicional de tumba y quema o se limpia a machete; en ocasiones, se aplica herbicida cuando está muy sucio con muchas malezas y hay poca mano de obra disponible. Mientras que los arroces de secano se siembran a chuzo, dejando caer algunas semillas en un orificio hecho con un palo de madera, los de agua se cultivan mediante el trasplante de las plántulas previamente germinadas en un semillero. El desyerbe y mantenimiento se hacen con machete y en el corte se usa la hoz. Por su robustez y resistencia a las condiciones ambientales, estos arroces no requieren abonos o venenos, lo cual reduce los costos de producción, el endeudamiento de los productores y la contaminación ambiental. El secado del grano por manojos que se cuelgan del techo donde reciben el calor del fogón facilita la labor y es más económico. Los hombres tienen mayor presencia en todo el ciclo productivo pero la familia o los vecinos participan según la necesidad. La demanda de mano de obra por el carácter manual de las distintas faenas es una fuente de empleo e ingreso para trabajadores sin tierra.

A diferencia de las semillas mejoradas que se compran en cada ciclo porque "se ponen débiles y ya no paren igual", las criollas se guardan después de cada temporada como "semilla de costal" para el consumo familiar, la venta o siembras futuras ${ }^{29}$. Así, en pequeña escala y sin la mediación de un proceso organizativo o un discurso que llame a una acción colectiva de resistencia contra las

El sector del arroz blanco está concentrado en pocas empresas que controlan desde la molinería hasta la comercialización, con poca regulación estatal. http://www.elespectador.com/ noticias/investigacion/el-arroz-de-discordia-articulo-568914.

En el marco de la firma del Tratado de Libre Comercio con Estados Unidos, el Gobierno nacional determinó los requisitos para la producción, acondicionamiento, importación, exportación, almacenamiento, comercialización y uso de semillas en el país mediante la Resolución 970 del 2010 del Instituto Colombiano Agropecuario. Esta resolución exige la siembra de semillas certificadas y registradas y restringe el uso y conservación de la "semilla de costal" solo para el autoconsumo y en áreas menores a cinco hectáreas. 
políticas agroalimentarias, el control de los agricultores sobre este patrimonio colectivo y de libre uso, circulación e intercambio les confiere un margen de seguridad y autonomía alimentaria frente a los monopolios de la industria semillera y biotecnológica.

Además de las ventajas económicas y agronómicas, la siembra de estas variedades "por tradición para la comida" tiene que ver con su sabor y textura y con las aspiraciones y expectativas de consumo: "son más sabrosos, cogen más la manteca y el grano es más largo. Abren y florean bien, quedan flojitos y suaves”. Parte del atractivo se debe al procesamiento del grano en el pilón, que solo remueve la cáscara más gruesa y deja el salvado y las vitaminas, minerales, proteína y fibra, por lo cual es más nutritivo y saciador, para comer "sin necesidad de liga y quedar satisfecho". Por todos estos atributos los criollos se denominan finos, a diferencia de los comerciales que se blanquean en el molino donde se cepillan y pulen para darles brillo, volverlos más cristalinos y "embellecerlos" para el mercado. De suerte que lo que le llega al consumidor, quien generalmente desconoce los procesos detrás del producto, es un grano blanco, suave, homogéneo, "puro" e "higiénico", que cumple con las expectativas comerciales, pero despojado de los nutrientes originales.

En relación con la desaparición de alimentos y variedades agrícolas tradicionales en su natal Grecia, Nadia Seremetakis (1994) señala que los cultivos y la comida son parte de las epistemologías locales y a su vez permiten una experiencia material de la historia. En el caso de los arroces criollos de La Mojana, estos hacen parte de la cultura material de producción y consumo regional, la identidad, la memoria social y la agencia de los agricultores. Son parte de lo que Kathleen Stewart (2007) llama los afectos ordinarios debido a la relación personal y afectiva que la gente establece con ellos. Al igual que en Japón (OhnukiTierney 1993) y África Occidental (Carney 2002), estos arroces son patrimonios materiales y culturales, resultado de la histórica interacción y mutua influencia entre agricultores, plantas, semillas y suelos en procesos de experimentación, adaptación y mejoramiento genético y agronómico en distintos ambientes. Incluso los nombres comunes y las narrativas que circulan en torno a ellos son referentes históricos, geográficos y sociales que confieren un sentido de lugar y memoria (tabla 1) (Feld y Basso 1996). 
Tabla 1. Nombres comunes de algunos arroces criollos cultivados en La Mojana

\begin{tabular}{l|l}
\multicolumn{1}{c|}{ Arroces criollos de secano: finos } & \multicolumn{1}{c}{ Arroces criollos de agua: chiles } \\
$\begin{array}{l}\text { Ina mono, ina blanco, marfil, marfil mono, } \\
\text { fortuna, rojo, palmira, palmira morado, palmira }\end{array}$ & $\begin{array}{l}\text { Chombo, chilimico, monito riaño, canilla, pedro } \\
\text { saco, maría la baja, pantalón sucio, comino, } \\
\text { ligerito, ligerito mono, ligerito blanco, ligerito } \\
\text { piedra, belepán, español, carretero, guayaquil, } \\
\text { pigua, mano negra, chejo negro, toldo sucio, diana palma ripiada, canelo, mariángela, } \\
\text { campeón }\end{array}$ \\
$\begin{array}{l}\text { criollo, piedra del cauca, maría la baja, mira mono, } \\
\text { blugones, guatapurí, rabo de vaca, cacao pelao, } \\
\text { blubonet }\end{array}$ & \\
\hline
\end{tabular}

Fuente: Elaboración propia a partir de entrevistas con agricultores.

Así, detrás de estas prácticas campesinas y la diversidad agroalimentaria, hay un acumulado cultural de observación, trabajo e ingenio que, si bien con frecuencia se representa como incompleto, atrasado e incluso irracional, se constituye en una práctica alternativa al sistema agroalimentario que ve en la tecnología y la ciencia soluciones genéricas a problemas complejos, como la seguridad alimentaria y nutricional y el cambio climático.

\section{Conclusiones}

La producción arrocera moderna en La Mojana se desarrolló en el contexto de una estructura inequitativa de la tenencia de la tierra preexistente, asociada con el acaparamiento y despojo de tierras y aguas públicas de uso comunal para la ganadería extensiva. El proceso del arroz no ha sido la principal causa de concentración de la tierra y los medios de vida en la región, pero sí es un factor parcial de dos tipos de acumulación. Por una parte, económica, a través de la lógica del mercado y los principios de eficiencia, rendimiento y productividad de quienes han tenido la tierra, la tecnología y el capital. Por otra, acumulación tóxica, en espacios, alimentos y cuerpos de productores y consumidores. Los efectos del despojo por contaminación son vastos y violentos, pero no siempre evidentes, porque suceden a través de procesos químicos, microbiológicos y micropolíticos que se difieren parcialmente en el tiempo, con lo cual son naturalizados en la vida cotidiana y entre las nuevas generaciones.

En la dinámica estudiada, los agentes y víctimas de la contaminación y el despojo son los mismos agricultores, pero quienes sufren los mayores daños en sus cuerpos y su salud son quienes manipulan los agroquímicos directamente: 
los productores más pequeños y los trabajadores agrícolas. Si bien ellos perciben y expresan algunos de sus efectos nocivos, no llegan a desarrollar un discurso estructurado o acciones colectivas contra el sistema que los produce ya que, en el ámbito local, el poder de la industria agroquímica global y de las tendencias de la acumulación capitalista se desdibuja por las mediaciones y escalas del mercado y por los beneficios directos de los insumos. No obstante, localmente y en pequeña escala, la agricultura comercial coexiste con modelos agroalimentarios más sostenibles que responden a racionalidades de diversificación económica, agronómica y alimentaria. A pesar de sus límites, el sistema del arroz criollo es un "espacio de posibilidades” (Goodman, Dupuis y Goodman 2011, 3) donde los mojaneros deciden autónomamente qué y cómo producir y consumir para su propio beneficio, bienestar y placer.

En una discusión sobre el despojo, Gillian Hart (2006) propone considerar sus especificidades históricas y geográficas para desnaturalizarlo y entenderlo como un proceso continuo. En el caso de La Mojana hemos contemplado la dimensión ambiental, cultural y corporal del despojo por contaminación, así como las percepciones y reacciones contradictorias que suscita la externalización de los costos y efectos socioambientales. Observar la contaminación como una forma de despojo contribuye a la comprensión de otros mecanismos extraeconómicos de acumulación de capital, que van más allá de la enajenación de la mano de obra o la privación de la tierra. Este es un tipo de despojo sutil, cotidiano y sostenido, en el que la naturaleza, los territorios rurales, los agricultores y los consumidores asumen los costos tóxicos de un sistema agroalimentario paradójico y a la vez perverso que, en nombre de la eficiencia, el bienestar, la seguridad alimentaria y la salud, degrada los recursos y socava las posibilidades de producción y reproducción social. En otras palabras, un sistema despojador de la vida misma.

\section{Referencias}

Aguilera, María. 2004. La Mojana: riqueza natural y potencial económico. Documentos de Trabajo sobre Economía Regional 48, octubre. Cartagena: Banco de la República.

Auyero, Javier y Débora Swistun. 2009. Flammable. Environmental Suffering in an Argentine Shantytown. Oxford: Oxford University Press.

Bellotti, Anthony, César Cardona y Stephen L. Lapointe. 1990. "Trends in Pesticide Use in Colombia and Brazil”. Journal of Agricultural Entomology 7 (3): 191-201. 
Borras, Saturnino, Ruth Hall, Ian Scoones, Ben White y Wendy Wolford. 2011. "Towards a Better Understanding of Global Land Grabbing”. The Journal of Peasant Studies 38 (2): 209-216.

Büscher, Bram. 2015. “The Ironies of Progress and Plunder: A Review of the Africa Progress Report 2014”. Development and Change 46 (4): 949-960.

Butler, Judith y Athena Athanasiou. 2013. Dispossession: The Performative in the Political. Cambridge: Polity Press.

Camacho, Juana. 2011. "Embodied Tastes: Food and Agrobiodiversity in the Colombian Andes”. Tesis doctoral en Antropología, Universidad de Georgia, Athens, Estados Unidos.

—. 2015. "Paisaje y patrimonio en La Mojana, Caribe colombiano”. Geografia Ensino \& Pesquisa (número especial) 19: 90-100.

Cárdenas, Omayda, Elizabeth Silva, Ligia Morales y Jaime Ortiz. 2005. "Estudio epidemiológico de exposición a plaguicidas organofosforados y carbamatos en siete departamentos colombianos, 1998-2001”. Biomédica 25: 170-180.

Cárdenas, Omayda, Elizabeth Silva y Jaime Eduardo Ortiz [q. e. p. d.]. 2010. "Uso de plaguicidas inhibidores de acetilcolinesterasa en once entidades territoriales de salud en Colombia, 2002-2005”. Biomédica 30: 95-106.

Cárdenas, Omayda, Elizabeth Silva y Gerardo Nava Tovar. 2012. "Actividad de la acetilcolinesterasa en trabajadores e individuos con riesgo de exposición a plaguicidas organofosforados y carbamatos en 15 departamentos de Colombia, 2006-2009”. Ciencia y Trabajo 14 (43): 120-128.

Carney, Judith. 2002. Black Rice: The African Origins of Rice Cultivation in the Americas. Cambridge: Harvard University Press.

Carolan, Michael. 2011. Embodied Food Politics. Burlington, VT: Ashgate Publishing.

Centro de Memoria Histórica (CMH). 2010. La tierra en disputa. Bogotá: Taurus.

Comisión Nacional de Reparación y Reconciliación (CNRR). 2009. El despojo de tierras y territorios. Aproximación conceptual. Bogotá: Kimpres.

Corpoica-INAT. "Producción agropecuaria sostenible en los ecosistemas de La Mojana”. Consultado el 17 de octubre del 2016. http://es.scribd.com/doc/111567530/Mojana-Corpoicaoctubre-23.

Corporación Observatorio del Mercado de Trabajo en Sucre (COMTS). 2013. "Diagnóstico del mercado laboral y perfil económico y productivo del departamento de Sucre”. Documento II.

De Angelis, Massimo. 2001. "Marx and Primitive Accumulation: The Continuous Character of Capital's 'Enclosures”. The Commoner 2. Consultado el 17 de octubre del 2016. http:// commoner.org.uk/02deangelis.pdf/.

Departamento Nacional de Planeación (DNP). 2009. Política nacional para la racionalización del componente de costos de producción asociado a los fertilizantes en el sector agropecuario. Conpes 3577. Bogotá: DNP. 
Departamento Nacional de Planeación (DNP) y Organización de las Naciones Unidas para la Agricultura y la Alimentación (FAO). 2003. Programa de desarrollo sostenible de la región de La Mojana. Bogotá: DNP.

Escobar, Arturo. 1995. Encountering Development: The Making and Unmaking of the Third World. Princeton: Princeton University Press.

Ezquerro-Cañete, Arturo. 2016. "Poisoned, Dispossessed and Excluded: A Critique of the Neoliberal Soy Regime in Paraguay”. Journal of Agrarian Change 16 (4): 702-710.

Fairhead, James, Melissa Leach e Ian Scoones. 2012. “Green Grabbing: A New Appropriation of Nature?”. The Journal of Peasant Studies 39 (2): 237-261.

Fals-Borda, Orlando. 2002. Historia doble de la costa. Resistencia en el San Jorge. Bogotá: Universidad Nacional de Colombia; Banco de la República; El Áncora.

Farquhar, Judith. 2002. Appetites: Food and Sex in Post-Socialist China. Durham y Londres: Duke University Press.

Feld, Steven y Keith Basso, eds. 1996. Senses of Place. Santa Fe: School of American Research Press.

Fernández, Daniel, Liliana Mancipe y Diana Fernández. 2010. “Intoxicación por organofosforados”. Revista Facultad de Medicina 18 (1): 84-92.

Flórez, Roicer Alberto. 2012. "Economía agrícola y circuitos comerciales en el estado soberano de Bolívar, 1857-1886”. Memorias, Revista Digital de Historia y Arqueología desde el Caribe Colombiano 9 (16): 131-164.

Garay, Luis Jorge, Fernando Barberi e Iván Cardona. 2012. Impactos del TLC con Estados Unidos sobre la economía campesina colombiana. Bogotá: Oxfam.

Goodman, David, Melanie Dupuis y Michael K. Goodman. 2011. Alternative Food Networks: Knowledge, Practice, and Politics. Londres: Routledge.

Hart, Gillian. 2006. "Denaturalizing Dispossession: Critical Ethnography in the Age of Resurgent Imperialism”. Antipode 38 (5): 977-1004.

Harvey, David. 2003. The New Imperialism. Oxford: Oxford University Press.

IARC-OMS. 2015. "Evaluation of Five Organophosphate Insecticides and Herbicides”. IARC Monographs. Lyon, Francia, 20 de marzo. http://www.iarc.fr/en/media-centre/iarcnews/ pdf/MonographVolume112.pdf.

Incoder. 2014. "Asignación de uso y manejo de los terrenos comunales de la Nación. Condiciones y procedimientos”. Ministerio de Agricultura. Incoder. Bogotá . https://www.google. com.co/webhp?sourceid=chrome-instant\&ion=1\&espv=2\&ie=UTF-8\#q=Incoder+2014+asig naci\%C3\%B3n+de+uso+y+manejo+de+los+terrenos+comunales+de+la+nacion\&* .

Instituto de Hidrología, Meteorología y Estudios Ambientales (Ideam). 2015. Estudio Nacional del Agua 2014. Bogotá: Ideam. http://documentacion.ideam.gov.co/openbiblio/bvirtual/023080/ENA_2014.pdf.

Instituto Nacional de Salud (INS). 2010. "Protocolo de vigilancia y control de intoxicaciones por plaguicidas”. Grupo de Vigilancia y Control en Salud Pública. Versión n. 00 
PRO-R02.003.0000.014. Bogotá. https://www.minsalud.gov.co/comunicadosPrensa/Documents/INTOXICACION_POR_PLAGUICIDAS.pdf.

—. 2016. "Vigilancia y análisis del riesgo en salud pública: protocolo de vigilancia en salud pública. Intoxicaciones por sustancias químicas”. Grupo Factores de Riesgo Ambiental Subdirección de Prevención Vigilancia y Control en Salud Pública. Instituto Nacional de Salud. Versión 02 PRO-R02.006. Bogotá. http://www.ins.gov.co/lineas-de-accion/Subdireccion-Vigilancia/sivigila/Protocolos\%20SIVIGILA/PRO\%20Intoxicaciones.pdf.

Kalmanovitz, Salomón y Enrique López E. 2002. Instituciones y desarrollo agrícola en Colombia a principios del siglo XX (parte II). Serie Borradores de Economía n. 224. Bogotá: Banco de la República.

Kay, Silvia y Jenny Franco. 2012. The Global Water Grab. A Primer. The Netherlands: The Transnational Institute (TNI).

Kloppenburg, Jack. 2010. "Impeding Dispossession, Enabling Repossession: Biological Open Source and the Recovery of Seed Sovereignty”. Journal of Agrarian Change 10 (3): 367-388.

Linebaugh, Peter. 2008. The Magna Carta Manifesto. Liberties and Commons for All. Berkeley: University of California Press.

Lock, Margaret y Vinh-Kim Nguyen. 2010. An Anthropology of Biomedicine. Oxford: WileyBlackwell.

Machado, Absalón. 2003. Ensayos sobre seguridad alimentaria. Bogotá: Universidad Nacional de Colombia.

Marín, Alexander. 2013. "Los dueños de los fertilizantes en Colombia”. Consultado el 17 de octubre del 2016. http://www.elespectador.com/noticias/investigacion/los-duenos-de-losfertilizantes-colombia-articulo-445007.

Martínez, Antonio. 2013. “Caracterización socioeconómica de los sistemas de producción de la región de La Mojana”. Revista Corpoica: Ciencia y Tecnología Agropecuaria 14 (2): 165-185.

Marx, Karl. (1867) 1976. Capital. A Critique of Political Economy, vol. 1. Nueva York: Penguin Books.

Mitman, Gregg, Michelle Murphy y Christopher Sellers, eds. 2004. Landscapes of Exposure: Knowledge and Illness in Modern Environments. Osiris 19. Chicago: Chicago University Press.

Ohnuki-Tierney, Emiko. 1993. Rice as Self: Japanese Identities through Time. Princeton: Princeton University Press.

Organización de las Naciones Unidas para la Alimentación y la Agricultura (FAO) y Organización Mundial de la Salud (OMS). 1985. Código Internacional de Conducta para la Gestión de Plaguicidas. Roma: FAO.

Organización Mundial de la Salud (OMS). 2003. "Directrices sobre la gestión de los plaguicidas para la salud pública. Informe de una consulta interregional”. Ciang Mai, Tailandia. Documento OMS: WHO/CDS/WHOPES/2003.7.

Organización Mundial de la Salud (OMS) y Organización de las Naciones Unidas para la Alimentación y la Agricultura (FAO). 2016. Joint FAO/WHO, Meeting on Pesticide Residues (JMPR). Ginebra, 9-13 de mayo. http://www.who.int/foodsafety/jmprsummary2016.pdf. 
Ostrom, Elinor. 2000. El gobierno de los bienes comunes. La evolución de las instituciones de acción colectiva. México D. F.: UNAM; CRIM; FCE.

Patel, Raj. 2008. Obesos y famélicos. El impacto de la globalización en el sistema alimentario mundial. Barcelona: Los Libros del Lince.

-. 2013. "The Long Green Revolution”. The Journal of Peasant Studies 40: (1): 1-63. DOI: 10.1080/03066150.2012.719224.

Perreault, Tom. 2013. "Dispossession by Accumulation? Mining, Water and the Nature of Enclosure on the Bolivian Altiplano”. Antipode 45 (5): 1050-1069.

Polanyi, Karl. (1944) 1991. La gran transformación. México D. F.: Fondo de Cultura Económica.

Pongutá, Basilio, Edineldo Lans-Ceballos y José Luis Barrera-Violeth. 2012. "Residuos de insecticidas organoclorados presentes en leche cruda comercializada en el departamento de Córdoba, Colombia”. Acta Agronómica 61 (1): 10-15. http://www.revistas.unal.edu.co/ index.php/acta_agronomica/article/view/32453/32845.

Posada Carbó, Eduardo. 1994. "Progreso y estancamiento: 1850-1950”. En Historia económica y social del Caribe colombiano, editado por Adolfo Meisel Roca, 231-284. Barranquilla: Ediciones Uninorte; ECOE.

-. 1998. El Caribe colombiano. Una historia regional (1870-1950). Bogotá: Banco de la República; El Áncora.

Quesada, James, Laurie Hart y Philippe Bourgois. 2011. "Structural Vulnerability and Health: Latino Migrant Laborers in the United States”. Medical Anthropology 30 (4): 339-362.

Ramírez, Bernardo y Edgar Rey. 1994. La Mojana. Poblamiento, producción y conflicto social. Cartagena: Costa Norte. Editores Colombia.

Ramírez, Jairo. 2012. “Caracterización sociodemográfica del área de desarrollo rural de La Mojana”. Informe presentado a la Subgerencia de Planificación e Información del Instituto Colombiano de Desarrollo Rural (Incoder). Bogotá. http://www.incoder.gov.co/documentos/Estrategia \%20de \%20Desarrollo \%20Rural/Pertiles \%20Territoriales/ADR \%20MOJANA/Perfil \%20Territorial/CARACTERIZACION \%20SOCIO-DEMOGRAFICA \%20LA \%20 MOJANA.pdf.

Reyes, Alejandro. 1978. Latifundio y poder político: la hacienda ganadera en Sucre. Bogotá: Cinep.

Saxton, Devra. 2014. "Strawberry Fields as Extreme Environments: The Ecobiopolitics of Farmworker Health”. Medical Anthropology 34 (2): 166-183. http://dx.doi.org/10.1080/01459 740.2014.959167.

Seremetakis, Nadia. 1994. The Senses Still. Chicago: University of Chicago Press.

Solano, Harold y José Luis Marrugo. 2014. "Plaguicidas organoclorados en leche de ganado vacuno de la zona arrocera del Hático, municipio de Fonseca, La Guajira”. Revista Alimentos Hoy 23 (33): 81-90.

Stewart, Kathleen. 2007. Ordinary Affects. Durham y Londres: Duke University Press.

Suárez, Aurelio. 2015. Efectos del TLC Colombia-EE. UU. sobre el agro: los rostros. Bogotá: Corcas Editores. 
-. 2007. El modelo agrícola colombiano y los alimentos en la globalización. Bogotá: Ediciones Aurora.

White, Ben, Saturnino M. Borras Jr., Ruth Hall, Ian Scoones y Wendy Wolford. 2012. "The New Enclosures: Critical Perspectives on Corporate Land Deals”. The Journal of Peasant Studies 39 (3-4): 619-647.

Zamosc, León. 1990. "Luchas campesinas y reforma agraria: la sierra ecuatoriana y la costa atlántica colombiana en perspectiva comparativa”. Revista Mexicana de Sociología 52 (2): 125-180.

Zibechi, Raúl y Michael Hardt. 2013. Preservar y compartir: bienes comunes y movimientos sociales. Buenos Aires: Mardulce. 